\title{
Overexpression of a Rosa rugosa Thunb. NUDX gene enhances biosynthesis of scent volatiles in petunia
}

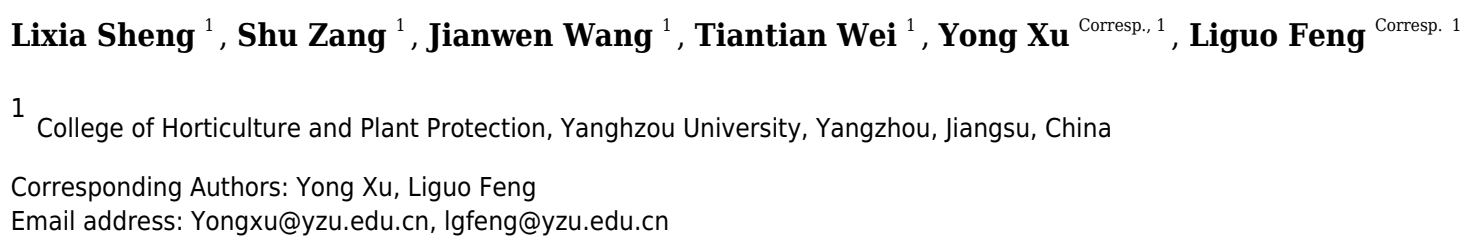

Rosa rugosa is an important natural perfume plant in China. Rose essential oil is known as 'liquid gold' and has high economic and health values. Monoterpenes are the main fragrant components of $R$. rugosa flower and essential oil. In this study, a member of the hydrolase gene family RrNUDX1 was cloned from Chinese traditional $R$. rugosa 'Tang Hong'. Combined analysis of RrNUDX1 gene expression and the aroma components in different development stages and different parts of flower organ, we found that the main aroma component content was consistent with the gene expression pattern. The RrNUDX1 overexpressed Petunia hybrida was acquired via Agrobacterium-mediated genetic transformation systems. The blades of the transgenic petunias became wider and its growth vigor became strong with stronger fragrance. Gas chromatography with mass spectrometry analysis showed that the contents of the main aroma components of the transgenic petunias including methyl benzoate significantly increased. These findings indicate that the RrNUDX1 gene play a role in enhancing the fragrance of petunia flowers. And it would lay an important foundation for the homeotic transformation of RrNUDXI in $R$. rugosa for cultivating new $R$. rugosa varieties of high-yield and -quality essential oil. 
1 Overexpression of a Rosa rugosa Thunb. NUDX gene 2 enhances biosynthesis of scent volatiles in petunia

3 Lixia Sheng, Shu Zang, Jianwen Wang, Yong $\mathrm{Xu}^{*}$, Liguo Feng*

4 College of Horticulture and Plant Protection, Yangzhou University, Yangzhou, Jiangsu, China;

5 Corresponding Author:

6 Yong Xu, Email address: Yongxu@yzu.edu.cn

7 Liguo Feng, Email address: lgfeng@yzu.edu.cn

8 


\section{Abstract}

Rosa rugosa is an important natural perfume plant in China. Rose essential oil is known as 'liquid gold' and has high economic and health values. Monoterpenes are the main fragrant components of $R$. rugosa flower and essential oil. In this study, a member of the hydrolase gene family RrNUDX1 was cloned from Chinese traditional R. rugosa 'Tang Hong'. Combined analysis of RrNUDX1 gene expression and the aroma components in different development stages and different parts of flower organ, we found that the main aroma component content was consistent with the gene expression pattern. The RrNUDX1 overexpressed Petunia hybrida was acquired via Agrobacterium-mediated genetic transformation systems. The blades of the transgenic petunias became wider and its growth vigor became strong with stronger fragrance. Gas chromatography with mass spectrometry analysis showed that the contents of the main aroma components of the transgenic petunias including methyl benzoate significantly increased. These findings indicate that the RrNUDX1 gene play a role in enhancing the fragrance of petunia flowers. And it would lay an important foundation for the homeotic transformation of RrNUDX1 in R. rugosa for cultivating new $R$. rugosa varieties of high-yield and -quality essential oil.

\section{Introduction}

Rosa rugosa Thunb., which usually serves as a good plant material for landscaping, is one of the oldest natural perfume plants. The rose essential oil extracted from its flowers is expensive and is mainly used in the high-end perfume, cosmetic, and health care industries (Ma et al., 2004). The demand for rose essential oil and its high fragrance quality is increasing in European and Asian markets. Besides optimization of extraction equipment and techniques, breeding R. rugosa variety rich in high-yield and -quality essential oil is the preferred way to overcome the high demand of rose essential oil.

Improving rose essential oil attributes by biotechnological breeding requires a well understanding of the biosynthesis of main aroma components in essential oil. Terpenes are the most abundant volatiles in floral aroma, including monoterpenes, sesquiterpenes and diterpenes, etc. (Pichersky \& Dudareva, 2007). In all aroma components of $R$. rugosa flowers, monoterpenes decide the content and quality of rose essential oil to a great extent. Monoterpenes such as citronellol, geraniol, nerol and their derivatives of acetate esters that account for $50 \%-70 \%$ of the overall mass fraction of rose essential oil are the main aroma components of rose essential oil (Feng et al., 2010; Magnard et al., 2015). Therefore, increasing monoterpenes synthesis in $R$. rugosa flowers would be an effective way to increase the content of aroma components in rose essential oil. The precursor of terpene isoprene pyrophosphate (IPP) was synthesized via the mevalonate (MVA) pathway in the cytoplasm and methylerythritol 4-phosphate (MEP) pathway in the plastid (Bick \& Lange, 2003; Sapir-Mir et al., 2008; Simkin et al., 2011; Lange \& Ahkami, 2013). Interestingly, the enzymatic machinery of geraniol biosynthesis in modern rose (Rosa $\times$ hybrida) is different with the classic pathway induced by a terpene synthase (Magnard et al., 2015). The gene Nucleoside diphosphates linked to some X moiety (NUDX1) play a key role in the fragrance formation revealed a unique pathway: The NUDX cuts down a phosphate group of substrate geranyl diphosphate (GPP) to produce geranyl monophosphate (GP) and then geraniol and other monoterpenes form from dephosphorylation of GP by an unknown phosphatase (Fig. S1) (Tholl \& Gershenzon, 2015).

The NUDX gene belongs to the Nudix hydrolase family and is widely found in various organisms, including bacteria, yeasts, algae, nematodes, vertebrates and plants (Bessman et al., 1996; Xu et al., 2004; Kraszewska, 2008). These enzyme families share a conserved Nudix motif $\mathrm{GX}_{5} \mathrm{EX}_{7}$ REVXEEXGU (Ogawa et al., 2008). Nudix hydrolase performs a role in regulation and signal transduction in plant stress (Bessman et al., 1996; Xu et al., 2004). It repairs the oxidative damage of guanine metabolism on DNA and protects the nucleic acid metabolic reactions in plants (Ogawa et al., 2009; Yoshimura \& Shigeoka, 2015). Recent studies on the Nudix hydrolase gene is mainly focused on the model plant Arabidopsis thaliana (L.) Heynh., which possesses a total of 29 Nudix hydrolase genes (Yoshimura \& Shigeoka, 2015). On the basis of 
55

predicted subcellular localization, the encoded proteins can be divided into three types: AtNUDX1 to -11, AtNUDX12 to -18, or AtNUDX19 to -24 (Ogawa et al., 2005; Yoshimura et al., 2007). In the AtNUDX gene family of Arabidopsis, overexpression of AtNUDX1 can attenuate or repair DNA and RNA oxidative damage (Yoshimura et al., 2007; Ogawa et al., 2008). Overexpression of AtNUDX2 gene could increase oxidative stress tolerance in Arabidopsis (Ogawa et al., 2009). As a positive regulatory protein in the signal transduction pathway of salicylic acid on which non-expressor of pathogenesis-related genes1 (NPR1) is dependent, AtNUDX6 significantly affects the plant immune response (Ishikawa et al., 2010). Meanwhile, the AtNUDX7 gene negatively regulates this pathway (Ishikawa et al., 2010).

In the present study, Chinese traditional R. rugosa was taken as a test material for cloning, subcellular localization, temporal and spatial expression analysis of RrNUDX1 gene which is the ortholog of RhNUDX1 (Fig. S1-S2, Magnard et al., 2015). By correlation analysis of main volatile monoterpenes in developmental stages and different parts of flower organs and transgene functional analysis, we prefer to investigate the function associated with volatile monoterpenes of $\operatorname{RrNUDX1}$ gene. The results could lay the foundation for understanding the mechanism of floral fragrance regulation and provide gene resources for creating rose germplasm with high essential oil.

\section{Materials \& Methods}

\section{Plant materials}

Rosa rugosa 'Tanghong', a representative Chinese traditional rose cultivar, was used as experimental material. The three-year-old clonal seedings planted in the field of Rose Resources of Yangzhou University (N32 $\left.23^{\prime} 27.64^{\prime \prime}, \mathrm{E} 119^{\circ} 25^{\prime} 10.23^{\prime \prime}\right)$ were selected as the flower source. According to previous division standard, the petals of flowers during five stages and different parts of full opening flowers were picked (Feng et al., 2014). For every sample, 3 biological replications were prepared with different ramets and 3-5 flowers of the same plant were selected for each replication.

\section{RNA extraction and purification}

Total RNA was isolated from different $R$. rugosa tissues according to the manufacturer's instructions of MiniBEST Universal RNA Extraction Kit (TaKaRa, Japan). RNA samples were treated with DNase using DNaseI kit (TaKaRa, Japan) according to the manufacturer's guidelines, and then quantified by a spectrophotometer (Eppendorf, Germany) at 230nm, 260nm and 280nm.

\section{Cloning of RrNUDX1 gene and Sequence analysis}

Full cDNA of RrNUDX1 gene was cloned by rapid amplification of cDNA end (RACE). The protocols of 3'RACE and 5'RACE were same as previously described in Feng et al. (2014) with the 3'RACE nest primers (5'-AGCCAAACCATCGCAGTA-3' for first round 5'-ATGGTTGGGGATGGTATG-3' for second round) and $5^{\prime}$ RACE gene-specific primer (5'-CTGCTGTGCCAATGCTGA-3'). The full-length cDNA of the RrNUDX1 gene was assembled and analyzed using DNAMAN 5.0 software.

\section{Subcellular localization of $\operatorname{RrNUDX1}$ gene}

The cDNA was synthesized from $1 \mu \mathrm{g}$ RNA using PrimeScript RT reagent Kit with gDNA Eraser (TaKaRa, Japan). Complete CDS of RrNUDX1 were isolated from the cDNA. CDS of RrNUDX1 and pBWA(V)HS-GFP vectors were digested by BsaI/Eco31I enzymes, and pBWA(V)HS-NUDX1-GFP fusion expression vector was constructed by using $\mathrm{T}_{4}$ DNA ligase connection, empty vector $\mathrm{pBWA}(\mathrm{V}) \mathrm{HS}-\mathrm{GFP}$ was used as control. The two vectors were transferred into the competent cells of Agrobacterium tumefaciens LBA4404 by electroporation in MicroPulser electroporator (Bio-RAD, USA) at voltage of $2.4 \mathrm{KV} / 5 \mathrm{~ms}$, and 
96 the positive bacteria were screened by Kanamycin and PCR. Then the screened positive bacteria were 97 expanded in LB liquid medium to $\mathrm{OD}_{600}=0.6$, and the lower epidermis of tobacco leaves was injected and 98 infected with these bacteria fluid, the tobacco plants were cultured in low light. After 2 days, tobacco leaves 99 were taken and the fluorescence of the transformed leaf cells was imaged using a confocal laser-scanning 100 microscope (Olympus FV10 ASW).

\section{Temporal and spatial expression analysis}

102 The relative expression of RrNUDX1 were analyzed by real-time quantitative reverse transcription 103 PCR (qRT-PCR). Rosa hybrid $\alpha$ - tubulin subunit actin gene (GenBank accession no. AF394915.1) was used 104 as an internal reference gene (Actin-F: 5'-GCCACCATCAAGACCAAG-3'; Actin-R: 5'105 ATCAATGCGGGAGAACAC-3'). Experiments of qPCR were performed with $12.5 \mu 1$ SYBR ${ }^{\circledR}$ Premix Ex Taq

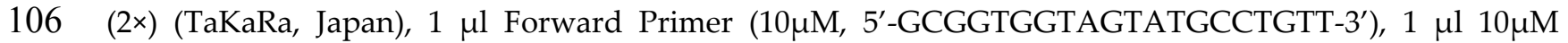
107 Reverse Primer (10 $\mu \mathrm{M}, 5^{\prime}$-TTCCTTCAGTTCCCTTGCTG-3'), $2 \mu \mathrm{l}$ cDNA and $8.5 \mu \mathrm{ldd} \mathrm{d}_{2} \mathrm{O}$. PCR procedure 108 is an initial incubation at $95^{\circ} \mathrm{C}$ for $5 \mathrm{~min}$, then followed by 40 cycles of $15 \mathrm{~s}$ at $95{ }^{\circ} \mathrm{C}, 30 \mathrm{~s}$ at $60{ }^{\circ} \mathrm{C}$, and $30 \mathrm{~s}$ at $10972{ }^{\circ} \mathrm{C}$ on a BIO-RAD CFX96 Real-Time System (Bio-Rad, USA). The expression level calculation according 110 to the comparative threshold cycle $(\mathrm{Ct})(2-\Delta \Delta \mathrm{Ct})$ method (Schmittgen \& Livak, 2008) followed previous 111 description (Feng et al., 2014).

\section{Gas chromatography with mass spectrometry (GC-MS) analysis of headspace volatiles}

$1131 \mathrm{~g}$ of fresh flower tissue and internal standard (3-nonanone, $0.8 \mu \mathrm{g} \cdot \mu \mathrm{L}^{-1}$, Sigma, USA) were used for 114 the headspace solide-phase microextraction for each sample. The extraction protocol and GC-MS protocol 115 were the same as our previous method with minor parameter adjustments (Feng et al., 2010; Feng et al., 116 2014). The adjustments included mass spectra scanning at $\mathrm{m} / \mathrm{z}$ 30-600 amu, more sensitive FFAP elastic 117 quartz capillary vessel column $(60 \mathrm{~m} \times 0.32 \mathrm{~mm}$ I.D., $1.0 \mu \mathrm{m}$ film, Agilent Corporation, USA) and column 118 temperature program (initial temperature at $50^{\circ} \mathrm{C}$ for $1 \mathrm{~min}$, and then increased at $5{ }^{\circ} \mathrm{C} / \mathrm{min}$ to $120^{\circ} \mathrm{C}$, then 119 increased at $8{ }^{\circ} \mathrm{C} / \mathrm{min}$ to $200{ }^{\circ} \mathrm{C}$, finally increased at $12{ }^{\circ} \mathrm{C} / \mathrm{min}$ to $250{ }^{\circ} \mathrm{C}$ which was maintained for $7 \mathrm{~min}$ ).

\section{Qualitative and quantitative analysis of headspace volatiles}

Significant data selection by the Xcalibur (a shareware of Thermo Electron Corporation, https://xcalibur.updatestar.com/) and quantitative analysis of the headspace compounds with internal standard 3-nonanone $\left(0.8 \mu \mathrm{g} \cdot \mu \mathrm{L}^{-1}\right)$ were performed as lab self-built method (Feng et al., 2010).

\section{Overexpression of RrNUDX1 gene in Petunia hybrida}

The recombinant pCAMBIA1304-RrNUDX1 expression vector construction and transfection into $A$. tumefaciens EHA105 competent cell (TransGen, China) followed previous method (Sheng et al., 2018). Transformation to Petunia hybrida cv 'Mitchell Diploid' via Agrobacterium-mediated infection on leaf disc were refers to Guo et al. (2014). Petunia explant leaf discs $(0.5 \mathrm{~cm}$ diameter) cut from 4 -week-old seedlings of aseptic seed were pre-cultured on pre-cultured MS medium ( $30 \mathrm{~g} / \mathrm{L}$ sucrose, $7 \mathrm{~g} / \mathrm{L}$ Agar, $3.0 \mathrm{mg} / \mathrm{L}$ 6-BA and $0.2 \mathrm{mg} / \mathrm{L} \mathrm{IAA}$ ) at $28{ }^{\circ} \mathrm{C}$ for $2 \mathrm{~d}$, then co-incubated with infection liquid where positive EHA105 (OD 600 0.8-1.0) was resuspended to $\mathrm{OD}_{600}=0.3$. After co-incubation for $5 \mathrm{~min}$, the explants with no residual infection liquid were cultured on pre-cultured medium with additional $30 \mu \mathrm{mol} / \mathrm{L}$ acetosyringone in dark for 2 days. Explants were transferred to the screening medium (pre-cultured medium with suited selection pressure) to induce callus and the medium was renewed every 2 weeks to subculture resistant calluses.

136

137 When the seedlings $(2-3 \mathrm{~cm}$ adventitious buds) regenerated from the callus, the buds were cut off to subculture on the rooting 1/2 MS medium (30 g/L sucrose, $7 \mathrm{~g} / \mathrm{L}$ agar, $0.1 \mathrm{mg} / \mathrm{L}$ NAA) with selection pressure. The hygromycin selection pressures were $7 \mathrm{mg} / \mathrm{L}$ and $6 \mathrm{mg} / \mathrm{L}$ at callus induction and buds 
138 rooting, respectively, and bacteriostat was $500 \mathrm{mg} / \mathrm{L}$ carbenicillin. The regeneration seedings growing up 139 to five leaves with one sprout were transplanted into soil. The culture condition was $16 \mathrm{~h}$ light period, light 140 intensity of $200 \mu \mathrm{mol} \mathrm{m} \mathrm{s}^{-1}$, temperature of $25^{\circ} \mathrm{C} / 23^{\circ} \mathrm{C}$ and relative humidity of $70 \%$. Callus GUS staining 141 and PCR were used to select the genomic transgenic petunia. The overexpression level of RrNUDX1 in

142 transgenic lines were detected by qRT-PCR. GC-MS was used to analyze the aroma composition and 143 content of wild-type and transgenic petunia flowers in bloom.

\section{Mathematics statistical methods}

145 The average value of three replicates with standard error (SE) was used as data of each sample. In 146 significance difference test between two sample, independent two-sample t-test was performed for two 147 data groups. In significance difference test of pairwise comparison of more than three samples, one-way 148 ANOVA (Analysis of Variance) and LSD (Least Significant Difference) was performed for multiple 149 comparison. All the calculation was based on the SPSS 18.0 (IBM SPSS Modeler 18.0, 150 https://www.ibm.com/support/pages/downloading-ibm-spss-modeler-180).

\section{Results}

\section{Isolation, sequence analysis and subcellular localization of RrNUDX1}

153 We successfully isolated a NUDX1 gene (RrNUDX1, GenBank accession number KX096710.1) related 154 to the monoterpenes biosynthesis of R. rugosa. The full cDNA of RrNUDX1 was 777 bp in length including 155 a $453 \mathrm{bp}$ coding sequence (CDS) which encodes 151 amino acids, the 5 ' untranslated region (UTR)(68 bp) 156 and the 3' UTR (224 bp). RrNUDX1 had high (98\%) sequence identity with homology gene of Rosa $\times$ hybrida 157 'Papa Meilland' (RhNUDX1, JQ820249.1) with only 7 SNPs including 3 nonsynonymous SNPs in the CDS. 158 RrNUDX1 protein had higher identity with homology protein of Rosa chinensis (AFW17224.1) (98.67\%) than 159 RhNUDX1 (M4I1C6.1) (98\%) (Fig. S2). RrNUDX1-GFP fusion protein exhibited no signal in the nucleus of 160 tobacco leaf cells, indicating that RrNUDX1 localized in the cytoplasm (Fig. 1).

\section{$161 \operatorname{RrNUDX1}$ expression and volatile monoterpenes accumulation in flower}

Temporal and spatial expression of RrNUDX1 had a significant difference from budding to senescence in R. rugosa 'Tanghong' (Fig. 2A-D). The expression level of RrNUDX1, which was low in the budding phase, rapidly increased from the early opening stage, reached the highest level at the half-opening stage, started to decrease from the blooming stage to $49.806 \%$ of that during the half-opening stage, and then markedly decreased during the senescence phase. The expression level of RrNUDX1 exhibited tissue specificity from the different organs of the flower, and the expression level reached the highest in the petals. However, the expression levels in the stamen and pistil were only $6.9 \%$ and $5.2 \%$ of that in the petals, respectively. Meanwhile, the expression levels in the anthocaulus, receptacle, and calyx were extremely low.

To verify the relations between the gene expression of RrNUDX1 and the accumulation of the main monoterpenoids in R. rugosa, we measured and determined the aroma component and content. Emphatical analysis was conducted on representative components, such as geraniol, citronellol, nerol (Fig. 2E-F), and their acetate derivatives, including geraniol acetate, citronellyl acetate, and neryl acetate (Fig. 2G-H). The contents of the main monoterpenes and their acetate derivatives initially increased and then decreased with

176 the flower development. Geraniol, nerol, geranyl acetate, and neryl acetate reached the highest levels in the 177 blooming stage, whereas citronellol and citronellyl acetate reached their highest levels during the half178 opening stage. The total content of the six main aromatic components in the half-opening stage was 10.12 $179 \mu \mathrm{g} / \mathrm{g}$ higher than that in the blooming stage. The six main aromatic components mainly originated from 180 the petal and stamen. The amount of the three monoterpene alcohols in the petal was more than four (4.34) 
181 times that in the stamen, whereas the amount of the three acetate derivatives in the stamen was $192.21 \mu \mathrm{g} / \mathrm{g}$,

182 which was more than three times that in the petal. Several aroma components were detected in the stamen,

183 whereas no monoterpene alcohols or acetate derivatives mentioned above were found in the anthocaulus,

184 receptacle, and calyx.

\section{Overexpression vector construction of RrNUDX1 gene and genetic transformation to petunia hybrida}

The petiole callus transformed by 35S: RrNUDX1 vector were stained with varying degrees of blue by GUS staining (Fig. S3). RrNUDX1 was successfully integrated into the genome of five petunia lines by PCR detection of six randomly selected lines (Fig. S4) and expressed significantly (Fig. S5). The wild-type and transgenetic petunia plants significantly differed in phenotype under the same conditions. Compared with those of the wild type, the leaves of the petunia with RrNUDX1 gene transformed were significantly wider, with $10.83 \mathrm{~mm}$ and $8.19 \mathrm{~mm}$ larger width and length than the wild type; moreover, the transgenic plants grows faster compared with the wild-type plants (Fig. 3, Table 1).

GC-MS were used to detect the aroma components of the petunia flowers in bloom, and 10 major aroma components were selected for statistical analysis (the sum of the 10 aroma components accounted for $80.10 \%$ of the total aroma components in petunia) (Table 2). Results showed that the contents of all ester fragrance ingredients, except for benzyl benzoate, increased in the transgenic petunia plants. In particular, the increase in the content of methyl benzoate was the most significantly. In the transgenic Line1, the content of methyl benzoate was $63.04 \mu \mathrm{g} / \mathrm{g}$, which was 1.69 times that in the wild-type plants. The alcohols were mainly benzyl alcohol and phenylethyl alcohol, and the contents of benzyl alcohol in transgenic Line1 and Line 2 were $25.58 \%$ and $53.49 \%$ higher than those in the wild-type plants. In addition, the contents of the two main phenolic compounds also increased, and the contents of isoeugenol in the three transgenic Lines were 3.2, 4.3, and 4.9 times those in the wild-type plants.

\section{Discussion}

Floral substances are secondary metabolites released from plant flowers, these substances are mainly composed of numerous low molecular-weight volatile compounds (Pichersky et al., 2006) that can attract insect pollination, improve the aesthetic value of ornamental plants, and enhance the quality and economic value of flower products (Negre-Zakharov et al., 2009). Geraniol is the main aroma component of Rosa rugosa flower and essential oil, it is formed in plants through two pathways. One is the MEP pathway in plastids, where upstream GPP was utilized by GES to form geraniol through catalysis and then forms nerol under isomerase action (Feng et al., 2014). The expression level of GES directly affects the yield of geraniol and consequently exerts a controlling effect on the synthesis of downstream indole monoterpenoid alkaloids (Kumar et al., 2015). Another special way to form geraniol is with the participation of NUDX1. NUDX1 and GES have similar functions in geraniol synthesis, both of them have the same precursor substance GPP, and can express in the cytoplasm for producing geraniol glycosides and geraniol (Magnard et al., 2015). In this study, $R r N U D X 1$ gene related to the biosynthesis of main aromatic components of Chinese traditional $R$. rugosa was investigated. Generally, the total aroma components during the bloom stage was consistent with RrNUDX1 expression, which initially increased and then decreased. Interestingly, the citronellol (and citronellyl acetate) reached the highest level in the half-opening stage unlike other components in the full opening stage and were more coincident with the RrNUDX1 mRNA accumulation than geraniol. The reason of unsynchronized peak time may be complex and we conjectured it should involve in other genes of monoterpene synthesis or conversion. On the one hand, the increasing geraniol (and its isomeride nerol) provide substrates of dehydrogenation for citronellol accumulation (Feng et al., 2014). One the other hand, potential GES of citronellol may decide on the citronellol accumulation on to a greater extent by inducing citronellol synthesis. Whereas, the studies of dehydrogenases of geraniol or nerol are greatly about geraniol acid and haven't identified the one for citronellol transformation until now 
226

227

228

229

230

231

232

233

234

235

236

237

238

239

240

241

242

243

244

245

246

247

248

249

250

251

252

253

254

255

256

257

258

259

260

261

262

263

264

265

266

267

268

(Hassan et al., 2012; Tan et al.; Tan et al., 2019), much less to the indistinguishable identification of geraniol or citronellol GES which is hidden in ambiguous function of terpenoid synthase family (Magnard et al., 2015). We prefer that the high citronellol accumulation more result from the induced response of an assumed GES. Though lack of strong evidence for our conjecture, our ongoing study found that several TPSS (GES candidates) with similar expression pattern with RrNUDX1 except in stamen and half opening stage while whether the induction would happen by these candidate GES is on testing (Unpublished). In addition, the aroma components of $R$. rugosa mainly concentrated in the petals, stamens, and pistils, and their contents were extremely low in the other parts, this is consistent with the expression of RrNUDX1 gene in different parts of floral organ. These results indicated that the expression of the RrNUDX1 gene are closely related the biosynthesis of monoterpene aromatic components in $R$. rugosa.

The faster growth morphology of overexpressed petunias couldn't be explained by fragrance synthetic function of RrNUDX1. NUDX1 belongs to the Nudix hydrolase gene family found in bacteria, viruses, and eukaryotes (Schmittgen \& Livak, 2008). NUDX1 protein of $A$. thaliana can effectively remove nucleotide damage due to cells ROS oxidation which increase tolerance to adverse environments (Ishibashi et al., 2005; Yoshimura \& Shigeoka, 2015) by hydrolyzing 8-oxo- (d) GTP, dNTP, NADH, and dihydroneopterin (Ishibashi et al., 2003; Klaus et al., 2005; Takagi et al., 2012). The transgenetic petunias with stronger growth vigor which is associated with the improved environment tolerance be owing to nucleotide damage repair ability of overexpressed RrNUDX1. Anyway, 68\% homology of RrNUDX1 with AtNUDX1 was just an clue of hydrolase function which need be confirmed by a follow-up study.

The petunia MD is an excellent material for verifying the function of floral genes (Verdonk et al., 2003), its aroma components are mainly composed of benzodiazepine components (Schuurink et al., 2006). Lvcker (Lücker et al., 2001) introduced the exogenous LIS gene into a non-scented petunia and found no direct effect on the production of the volatile linalool, and liquid chromatogram test showed the presence of linalool actually in the form of glycosides. In the present study, overexpression of the RrNUDX1 gene in petunia MD did not influence the type of volatile fragrance components of petunia. The reason for this result is probably that the RrNUDX1 gene is mainly located in the MVA and MEP pathways, therefore, the petunia Benzene/phenylpropanoid metabolites cannot be directly used as a substrates (Lücker et al., 2001). However, overexpression of RrNUDX1 gene effectively improved the accumulation of major aromatic components such as methyl benzoate in petunia flowers. These findings indicate that the RrNUDX1 gene from traditional Chinese $R$. rugosa has the function of enhancing the fragrance of petunia flowers. And these results lay an important foundation for the homeotic transformation of RrNUDX1 in R. rugosa for cultivating new rose varieties with high essential oil content.

\section{Conclusions}

We identified the gene RrNUDX1 from R. rugosa 'Tang Hong' whose expression pattern was correlated with the main aroma component content. The heterologously overexpressed Petunia hybrida have stronger growth vigor with wider blades and stronger fragrance with significantly increasing methyl benzoate. These findings indicate that the RrNUDX1 gene play a role in enhancing the fragrance of petunia flowers. Further study of regulation of RrNUDX1 would provide excellent genetic resources and technical means for the aroma quality improvement of other flowers.

\section{References:}

Bessman, M.J., Frick, D.N., and O'Handley, S.F. 1996. The MutT proteins or “Nudix” hydrolases, a family of versatile, widely distributed, "housecleaning" enzymes. JOURNAL OF BIOLOGICAL CHEMISTRY 271:2505925062 .

Peer) reviewing PDF | (2020:11:55499:1:1:NEW 15 Feb 2021) 
269 Bick, J.A., and Lange, B.M. 2003. Metabolic cross talk between cytosolic and plastidial pathways of isoprenoid 270 biosynthesis: unidirectional transport of intermediates across the chloroplast envelope membrane. ARCHIVES OF 271 BIOCHEMISTRY AND BIOPHYSICS 415:146-154.

272 Feng, L.G., Chen, C., Sheng, L.X., Liu, P., Tao, J., Su, J.L., and Zhao, L.Y. 2010. Comparative analysis of headspace 273 volatiles of Chinese Rosa rugosa. MOLECULES 15:8390-8399.

274 Feng, L., Chen, C., Li, T., Wang, M., Tao, J., Zhao, D., and Sheng, L. 2014. Flowery odor formation revealed by 275 differential expression of monoterpene biosynthetic genes and monoterpene accumulation in rose (Rosa rugosa 276 Thunb.). PLANT PHYSIOLOGY AND BIOCHEMISTRY 75:80-88.

277 Guo, Y.L., Yu, Y., Yang, Z., Qin, X.T., Ma, J., Han, Y., Yang, X., and Li, M.Y. 2014. Over-expressing PMADS20-

278 SRDX repressor leads to the formation of ectopic trichome and stoma on petals and pistils in petunia. ACTA HORTIC. 279 SIN 41:509-520.

280 Hassan, M., Maarof, N.D., Ali, Z.M., Noor, N.M., Othman, R., and Mori, N. 2012. Monoterpene alcohol metabolism: identification, purification, and characterization of two geraniol dehydrogenase isoenzymes from Polygonum minus leaves. JOURNAL OF THE AGRICULTURAL CHEMICAL SOCIETY OF JAPAN 76:1463-1470.

283 Ishibashi, T., Hayakawa, H., Ito, R., Miyazawa, M., Yamagata, Y., and Sekiguchi, M. 2005. Mammalian enzymes for preventing transcriptional errors caused by oxidative damage. NUCLEIC ACIDS RESEARCH 33:3779-3784. Ishibashi, T., Hayakawa, H., and Sekiguchi, M. 2003. A novel mechanism for preventing mutations caused by oxidation of guanine nucleotides. EMBO REPORTS 4:479-483. Ishikawa, K., Yoshimura, K., Harada, K., Fukusaki, E., Ogawa, T., Tamoi, M., and Shigeoka, S. 2010. AtNUDX6, an ADP-ribose/NADH pyrophosphohydrolase in Arabidopsis, positively regulates NPR1-dependent salicylic acid signaling. PLANT PHYSIOLOGY 152:2000-2012.

Ishikawa, K., Yoshimura, K., Ogawa, T., and Shigeoka, S. 2010. Distinct regulation of Arabidopsis ADPribose/NADH pyrophosphohydrolases, AtNUDX6 and 7, in biotic and abiotic stress responses. PLANT SIGNALING \& BEHAVIOR 5:839-841.

293 Klaus, S.M., Wegkamp, A., Sybesma, W., Hugenholtz, J., Gregory, J.F., and Hanson, A.D. 2005. A nudix enzyme removes pyrophosphate from dihydroneopterin triphosphate in the folate synthesis pathway of bacteria and plants. JOURNAL OF BIOLOGICAL CHEMISTRY 280:5274-5280.

296 Kraszewska, E. 2008. The plant Nudix hydrolase family. ACTA BIOCHIMICA POLONICA 55:663-671.

297 Kumar, K., Kumar, S.R., Dwivedi, V., Rai, A., Shukla, A.K., Shanker, K., and Nagegowda, D.A. 2015. Precursor 298 feeding studies and molecular characterization of geraniol synthase establish the limiting role of geraniol in 299 monoterpene indole alkaloid biosynthesis in Catharanthus roseus leaves. PLANT SCIENCE 239:56-66.

300 Lange, B.M., and Ahkami, A. 2013. Metabolic engineering of plant monoterpenes, sesquiterpenes and diterpenes301 current status and future opportunities. PLANT BIOTECHNOLOGY JOURNAL 11:169-196.

302 Lücker, J., Bouwmeester, H.J., Schwab, W., Blaas, J., Van Der Plas, L.H., and Verhoeven, H.A. 2001. Expression of 303 Clarkia S-linalool synthase in transgenic petunia plants results in the accumulation of 304 S - linalyl - $\beta$ - d - glucopyranoside. THE PLANT JOURNAL 27:315-324.

305 Ma, X.H., Wang, Y.H., Wei, Q., and Zhang, G.J. 2004. Study on processing technology of rose essential oil. 306 CHEMISTRY AND INDUSTRY OF FOREST PRODUCTS 24:80-84.

307 Magnard, J., Roccia, A., Caissard, J., Vergne, P., Sun, P., Hecquet, R., Dubois, A., Hibrand-Saint Oyant, L., Jullien, 
308 F., and Nicolè, F. 2015. Biosynthesis of monoterpene scent compounds in roses. SCIENCE 349:81-83.

309 Negre-Zakharov, F., Long, M.C., and Dudareva, N. 2009. Floral scents and fruit aromas inspired by nature. Plant-

310 derived Natural Products. Springer, 405-431.

311 Ogawa, T., Ishikawa, K., Harada, K., Fukusaki, E., Yoshimura, K., and Shigeoka, S. 2009. Overexpression of an

312 ADP - ribose pyrophosphatase, AtNUDX2, confers enhanced tolerance to oxidative stress in Arabidopsis plants. The

313 Plant Journal 57:289-301.

314 Ogawa, T., Ueda, Y., Yoshimura, K., and Shigeoka, S. 2005. Comprehensive analysis of cytosolic Nudix hydrolases

315 in Arabidopsis thaliana. JOURNAL OF BIOLOGICAL CHEMISTRY 280:25277-25283.

316 Ogawa, T., Yoshimura, K., Miyake, H., Ishikawa, K., Ito, D., Tanabe, N., and Shigeoka, S. 2008. Molecular

317 characterization of organelle-type Nudix hydrolases in Arabidopsis. PLANT PHYSIOLOGY 148:1412-1424.

318 Ogawa, T., Yoshimura, K., and Shigeoka, S. 2008. Functional analysis of an 8-oxo-7, 8-dihydro-2'-deoxyguanosine

319 5'-triphosphate pyrophosphohydrolase, AtNUDX1, involved in repair of oxidative DNA damage in Arabidopsis

320 thaliana. Photosynthesis. Energy from the Sun. Springer, 1323-1326.

321 Pichersky, E., Noel, J.P., and Dudareva, N. 2006. Biosynthesis of plant volatiles: nature's diversity and ingenuity.

322 SCIENCE 311:808-811.

323 Pichersky, E., and Dudareva, N. 2007. Scent engineering: toward the goal of controlling how flowers smell. TRENDS

324 IN BIOTECHNOLOGY 25:105-110.

325 Sapir-Mir, M., Mett, A., Belausov, E., Tal-Meshulam, S., Frydman, A., Gidoni, D., and Eyal, Y. 2008. Peroxisomal

326 localization of Arabidopsis isopentenyl diphosphate isomerases suggests that part of the plant isoprenoid mevalonic

327 acid pathway is compartmentalized to peroxisomes. PLANT PHYSIOLOGY 148:1219-1228.

328 Schmittgen, T.D., and Livak, K.J. 2008. Analyzing real-time PCR data by the comparative C T method. NATURE

329 PROTOCOLS 3:1101.

330 Schuurink, R.C., Haring, M.A., and Clark, D.G. 2006. Regulation of volatile benzenoid biosynthesis in petunia 331 flowers. TRENDS IN PLANT SCIENCE 11:20-25.

332 Sheng, L., Zeng, Y., Wei, T., Zhu, M., Fang, X., Yuan, X., Luo, Y., and Feng, L. 2018. Cloning and Functional 333 Verification of Genes Related to 2-Phenylethanol Biosynthesis in Rosa rugosa. GENES 9:576.

334 Simkin, A.J., Guirimand, G., Papon, N., Courdavault, V., Thabet, I., Ginis, O., Bouzid, S., Giglioli-Guivarc, N., and 335 Clastre, M. 2011. Peroxisomal localisation of the final steps of the mevalonic acid pathway in planta. PLANTA $336234: 903$.

337 Takagi, Y., Setoyama, D., Ito, R., Kamiya, H., Yamagata, Y., and Sekiguchi, M. 2012. Human MTH3 (NUDT18) 338 protein hydrolyzes oxidized forms of guanosine and deoxyguanosine diphosphates comparison with MTH1 and 339 MTH2. JOURNAL OF BIOLOGICAL CHEMISTRY 287:21541-21549.

340 Tan, C.S., Abd-Hamid, N., Chew, J.K., Hassan, M., Ismail, I., Ng, C.L., and Zainal, Z. 2019. Molecular 341 characterisation of nerol dehydrogenase gene $(\mathrm{PmNeDH})$ from Persicaria minor in response to stress-related 342 phytohormones. JOURNAL OF PLANT INTERACTIONS 14:424-431. 10.1080/17429145.2019.1638457

343 Tan, C.S., Abd-Hamid, N., Chew, J.K., Hassan, M., Ismail, I., Ng, C.L., and Zainal, Z. 2019. Molecular

344 characterisation of nerol dehydrogenase gene $(\mathrm{PmNeDH})$ from Persicaria minor in response to stress-related 345 phytohormones. JOURNAL OF PLANT INTERACTIONS 14:424-431.

346 Tholl, D., and Gershenzon, J. 2015. The flowering of a new scent pathway in rose. SCIENCE 349:28-29. 
347 Verdonk, J.C., De Vos, C.R., Verhoeven, H.A., Haring, M.A., van Tunen, A.J., and Schuurink, R.C. 2003. Regulation

348 of floral scent production in petunia revealed by targeted metabolomics. PHYTOCHEMISTRY 62:997-1008.

349 Xu, W., Dunn, C.A., Jones, C.R., D'Souza, G., and Bessman, M.J. 2004. The 26 Nudix hydrolases of Bacillus cereus, 350 a close relative of Bacillus anthracis. JOURNAL OF BIOLOGICAL CHEMISTRY 279:24861-24865.

351 Yoshimura, K., Ogawa, T., Ueda, Y., and Shigeoka, S. 2007. AtNUDX1, an 8-oxo-7, 8-dihydro-2' -deoxyguanosine 352 5'-triphosphate pyrophosphohydrolase, is responsible for eliminating oxidized nucleotides in Arabidopsis. PLANT 353 AND CELL PHYSIOLOGY 48:1438-1449.

354 Yoshimura, K., and Shigeoka, S. 2015. Versatile physiological functions of the Nudix hydrolase family in 355 Arabidopsis. BIOSCIENCE, BIOTECHNOLOGY, AND BIOCHEMISTRY 79:354-366. 


\section{Figure 1}

Figure 1 Subcellular localization of RrNUDX1 .

Fluorescence signals were visualized using confocal laser-scanning microscopy. Green fluorescence indicates GFP, red fluorescence indicates chloroplast autofluorescence (a) 35S: RrNUDX1-GFP; (b) 35S: GFP.

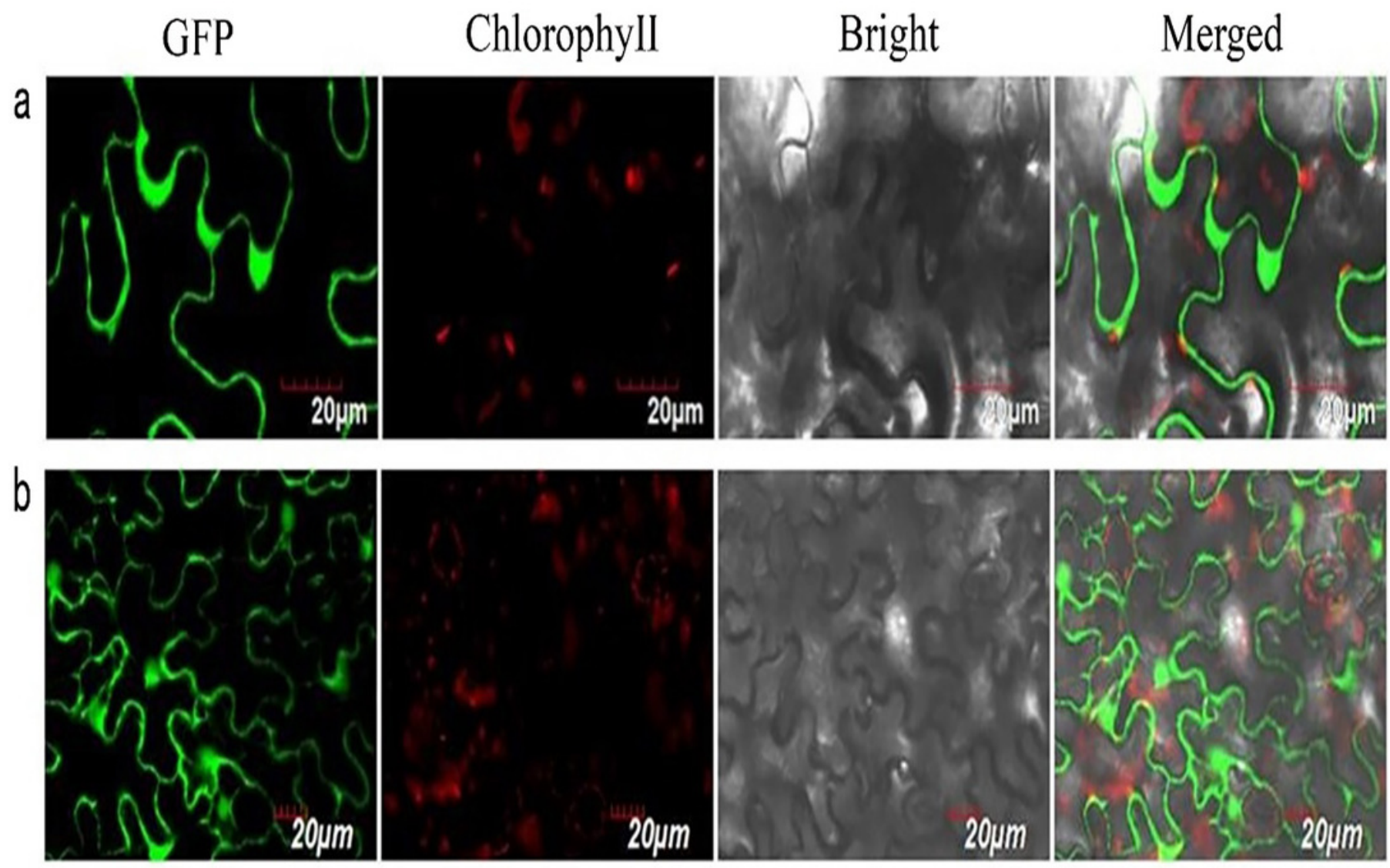




\section{Figure 2}

Figure 2 Expression of RrNUDXI and major volatile components in different development stages (A) and different parts of flower organ (B) in R. rugosa 'Tanghong'.

(C) and (D) Relative expression level of RrNUDX1; (E)-(H) Major volatile components. S1: budding stage, S2: early opening stage, S3: half opening stage, S4: full opening stage, S5: withering stage; P1: petal, P2: stamen, P3: pistil, P4: calyx, P5: receptacle, P6: pedicle. Values represent the means $\pm S E$. Letters $(a, b, c)$ stand for significantly different (LSD test, $P<$ 0.05). 
A

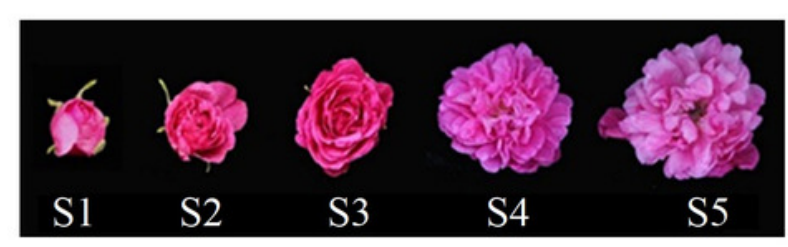

C

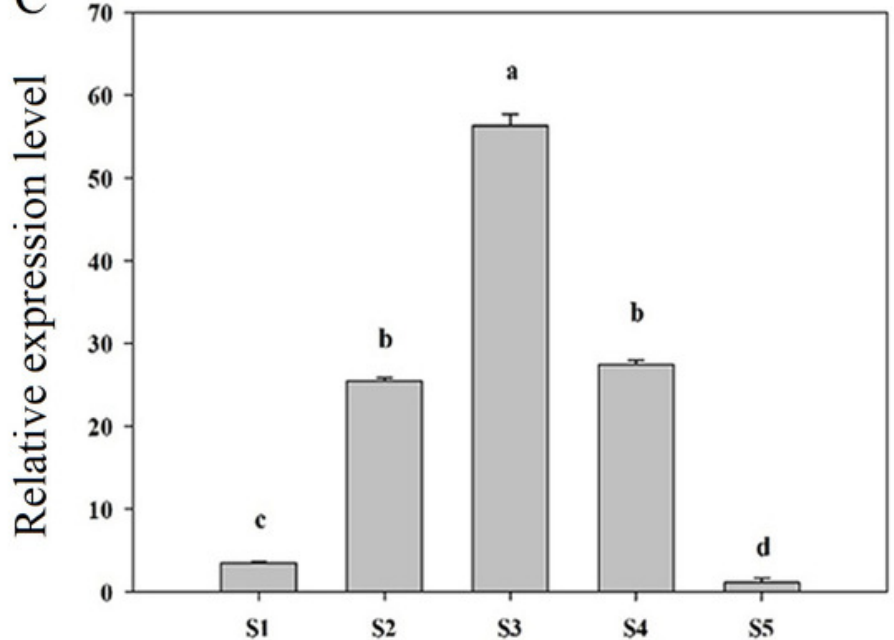

E 256 口 Citronellol

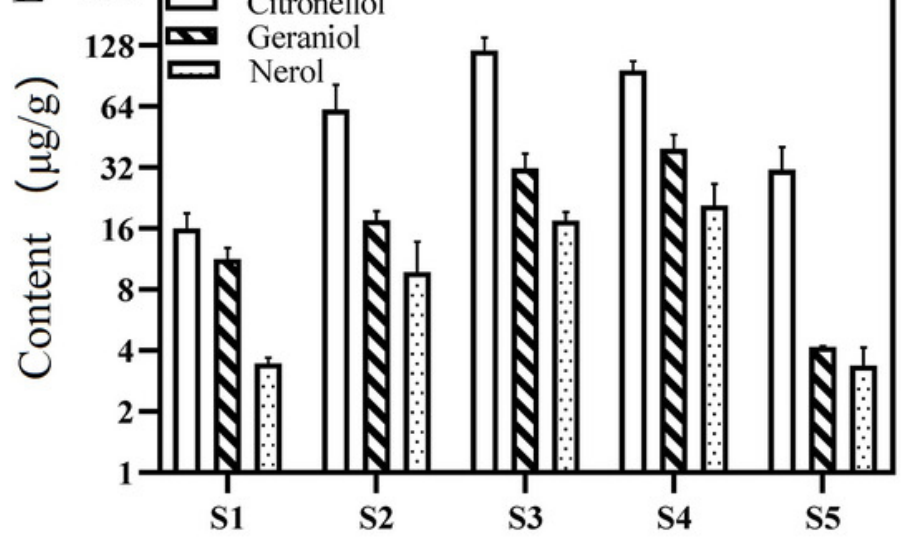

G

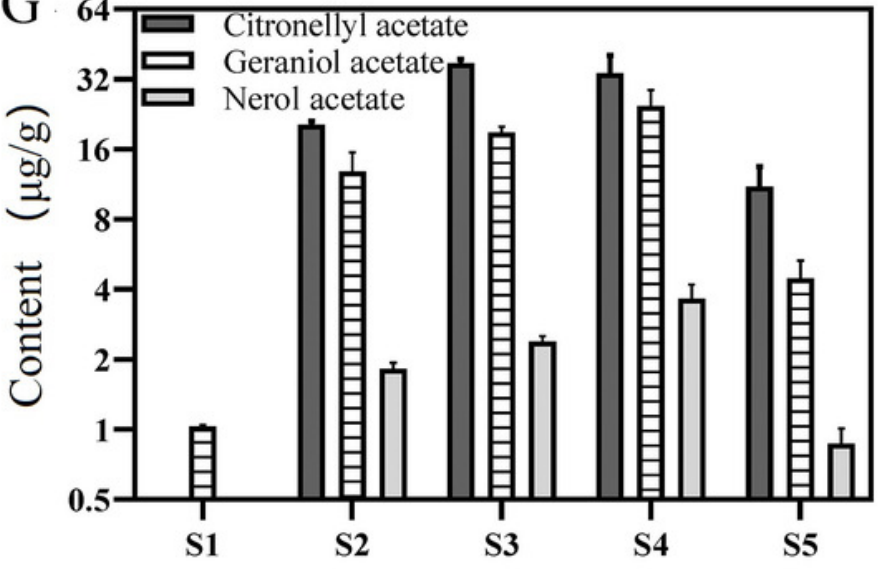

B

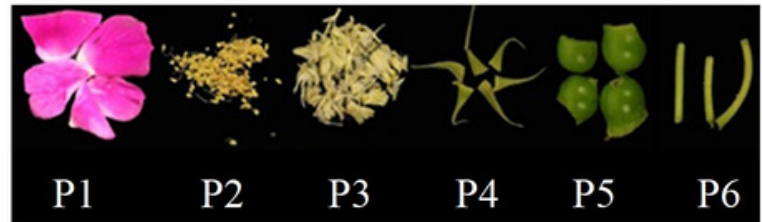

D

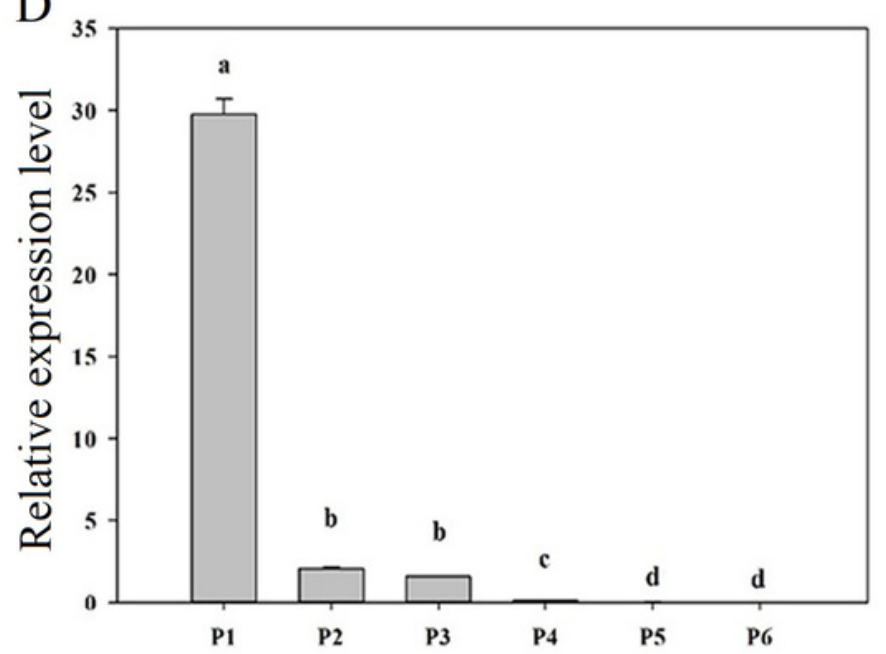

$\mathrm{F}$
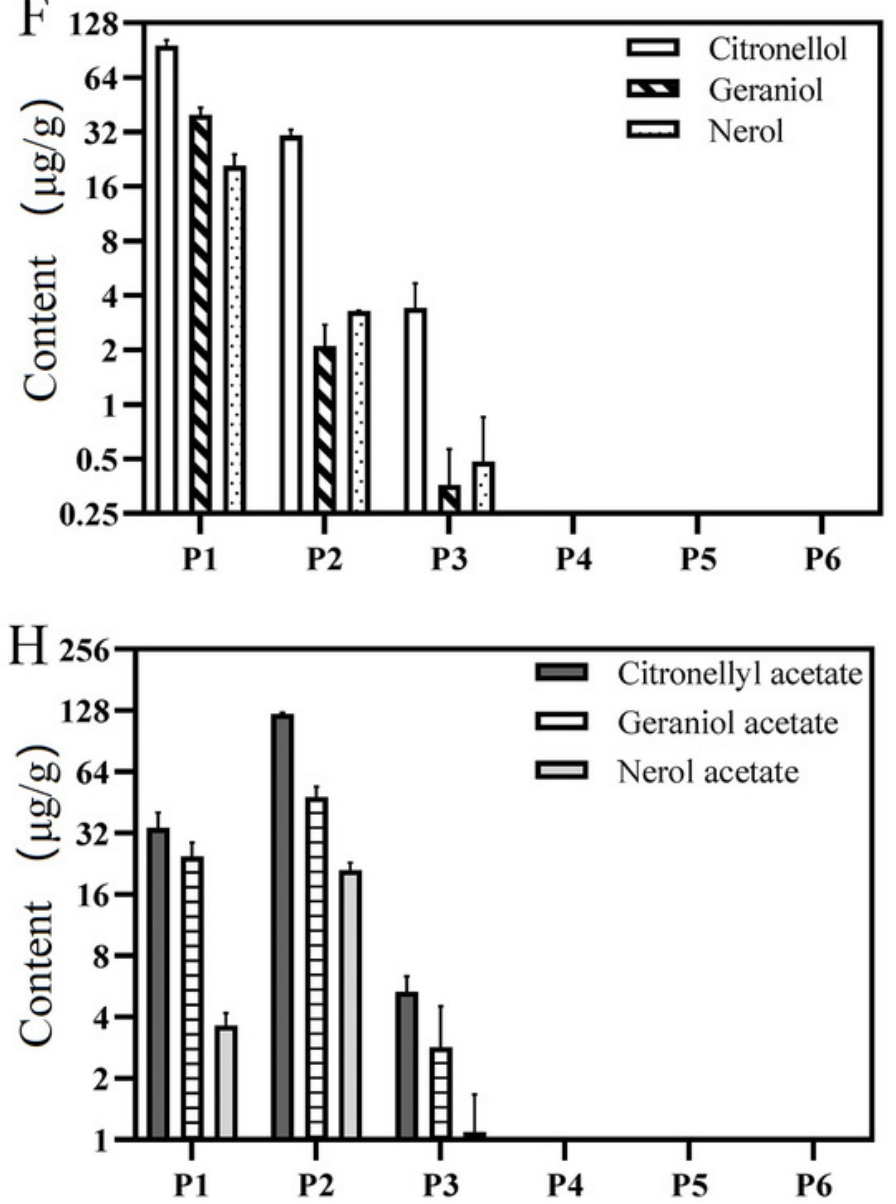


\section{Figure 3}

Figure 3 Phenotype of transgenic petunia hybrida.

WT1-2: Wild type; RrNUDX11-3: Transgenic petunia Line1, line2 and Line3. (a) Phenotype of wild and transgenic petunia plants after 35 days of transplanting; (b) A wild type petunia under flowering and a transgenic petunia under flowering; (c) Leaf morphology of wild and transgenic petunia plants after 35 days of transplanting.

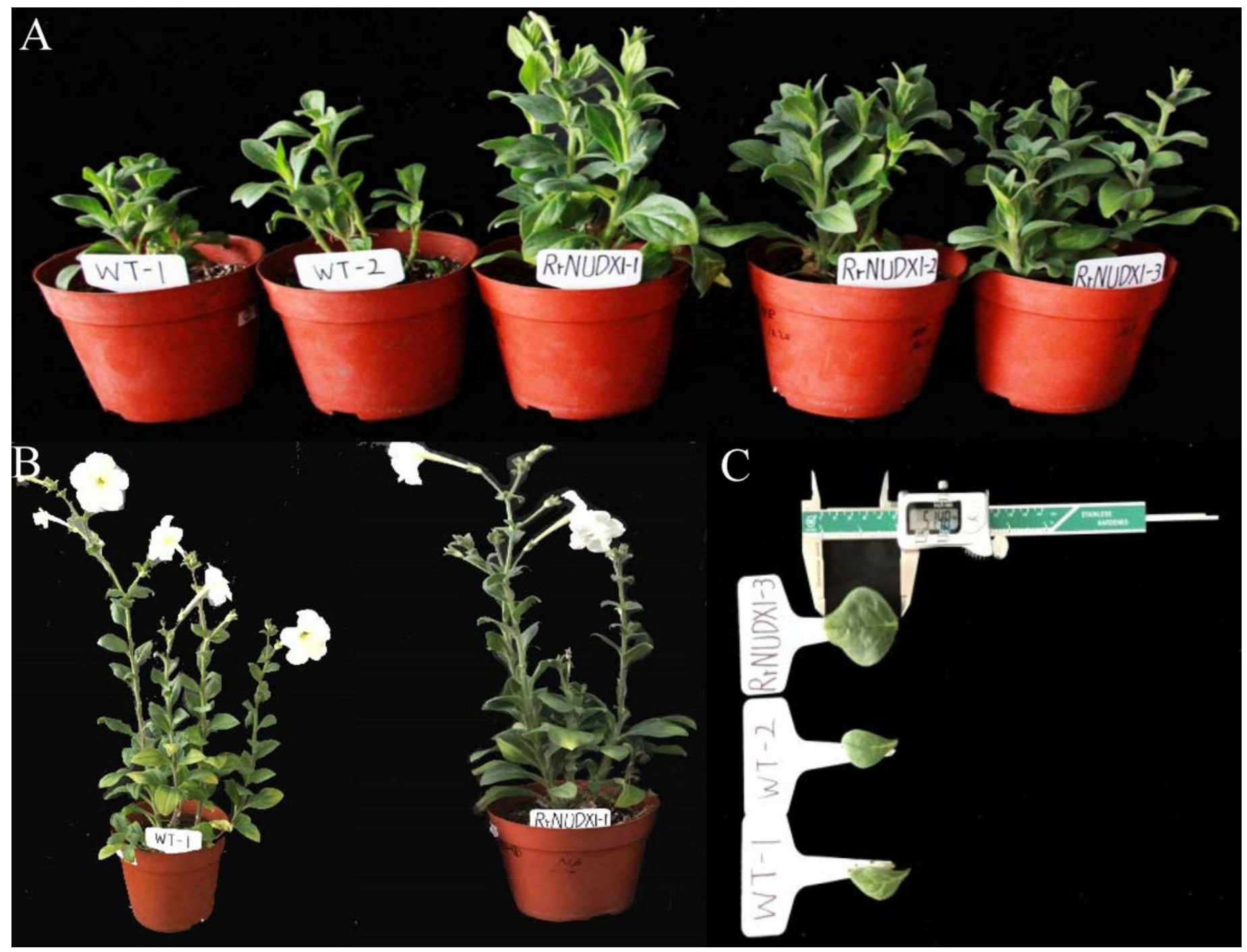




\section{Table $\mathbf{1}$ (on next page)}

Comparison of leaf morphology between wild and transgenic plantsafter 35 days of transplanting 
1 Table 1. Comparison of leaf morphology between wild and transgenic plantsafter 35 days of transplanting

\begin{tabular}{ccc}
\hline Index & $\begin{array}{c}\text { Wild type } \\
(\mathrm{mm})\end{array}$ & $\begin{array}{c}\text { Transgenetic plants } \\
(\mathrm{mm})\end{array}$ \\
\hline Leaf length & $36.42 \pm 0.99 \mathrm{~b}$ & $47.25 \pm 4.49 \mathrm{a}$ \\
Leaf width & $17.39 \pm 1.34 \mathrm{~b}$ & $25.58 \pm 6.45 \mathrm{a}$ \\
Aspect ratio & $2.1 \pm 0.11 \mathrm{a}$ & $1.84 \pm 0.13 \mathrm{~b}$ \\
\hline
\end{tabular}

2 Values represent the means \pm SE. Different letters $(a, b, c)$ stand for significantly different $($ LSD test, P $<0.05)$

3 
Table 2 (on next page)

Comparative analysis of floral components in wild type and transgenic petunia 
Table 2. Comparative analysis of floral components in wild type and transgenic petunia

\begin{tabular}{ccccc}
\hline \multirow{2}{*}{ Components } & $W T$ & Line1 & Line2 & Line3 \\
\cline { 2 - 5 } & $0.08 \pm 0.03 \mathrm{c}$ & $0.18 \pm 0.07 \mathrm{~b}$ & $0.18 \pm 0.01 \mathrm{~b}$ & $0.25 \pm 0.03 \mathrm{a}$ \\
\hline Tetradecane & $37.32 \pm 0.86 \mathrm{~b}$ & $63.04 \pm 2.22 \mathrm{a}$ & $52.24 \pm 11.66 \mathrm{a}$ & $39.53 \pm 1.02 \mathrm{~b}$ \\
Methyl benzoate & $0.13 \pm 0.02 \mathrm{~b}$ & $0.20 \pm 0.02 \mathrm{a}$ & $0.21 \pm 0.03 \mathrm{a}$ & $0.11 \pm 0.03 \mathrm{~b}$ \\
Phenylmethyl & $0.21 \pm 0.03 \mathrm{~b}$ & $0.35 \pm 0.07 \mathrm{a}$ & $0.31 \pm 0.03 \mathrm{a}$ & $0.30 \pm 0.07 \mathrm{a}$ \\
acetate & $0.24 \pm 0.01 \mathrm{~b}$ & $0.67 \pm 0.18 \mathrm{a}$ & $0.64 \pm 0.39 \mathrm{a}$ & $0.25 \pm 0.00 \mathrm{~b}$ \\
Methyl salicylate & $5.94 \pm 1.91 \mathrm{ab}$ & $8.91 \pm 1.22 \mathrm{a}$ & $5.23 \pm 3.42 \mathrm{~b}$ & $3.79 \pm 0.08 \mathrm{~b}$ \\
Benzyl butyrate & $0.43 \pm 0.21 \mathrm{a}$ & $0.54 \pm 0.13 \mathrm{a}$ & $0.66 \pm 0.38 \mathrm{a}$ & $0.33 \pm 0.14 \mathrm{a}$ \\
Benzyl benzoate & $0.41 \pm 0.01 \mathrm{a}$ & $0.57 \pm 0.03 \mathrm{a}$ & $0.43 \pm 0.29 \mathrm{a}$ & $0.28 \pm 0.18 \mathrm{a}$ \\
Benzyl alcohol & $0.35 \pm 0.06 \mathrm{~b}$ & $0.98 \pm 0.34 \mathrm{a}$ & $0.50 \pm 0.31 \mathrm{~b}$ & $0.44 \pm 0.03 \mathrm{~b}$ \\
Phenethyl alcohol & $0.10 \pm 0.04 \mathrm{c}$ & $0.32 \pm 0.06 \mathrm{~b}$ & $0.43 \pm 0.08 \mathrm{a}$ & $0.49 \pm 0.10 \mathrm{a}$ \\
\hline Eugenol & &
\end{tabular}

2 Values represent the means \pm SE. Different letters $(a, b, c)$ stand for significantly different (LSD 3 test, $\mathrm{P}<0.05)$. 